\title{
Capabilities of a New Sample Preparation Method for Probing Polymer Penetration and Localization into Wood Without Embedding
}

\author{
Brian Dorvel $^{1}$, Praveenkumar Boopalachandran ${ }^{1}$, Andrew Bowling $^{2}$, Xue Chen $^{3}$, and Steve King ${ }^{3}$ \\ ${ }^{1}$ Dow Chemical Company, Core R\&D- Division of Analytical Sciences, Freeport, TX, U.S.A 77541 \\ ${ }^{2}$ Dow Agrosciences, Advanced Technology Development, Indianapolis, IN, U.S.A 46268 \\ ${ }^{3}$ Dow Chemical Company, Industrial Solutions R\&D, Freeport, TX, U.S.A 77541
}

The changes in the wood structure after modification have been an interest in the wood industry for wood treaters, biologists, and botanists. However, due to the complex wood structure, it is always a challenge to obtain a high quality image of the modified wood with high resolution, due to the wood cutting, polishing, and staining methods. A description of the results using this new preparation technique to observe and measure polymer penetration and morphology in the wood will be presented.

Typically, the wood needs to be embedded since it is porous and tough to cross section without fixing the polymer in place, but several polymers are miscible in typical embedding media and may disrupt the localization of the polymer modified wood. Razor blades or normal diamond microtomy knives for sample preparation can also cause sample damage and compression, affecting the resolution. To minimize compression in biological samples, several methods have been employed but mainly using vitreous ice or cryomicrotomy [1][2]. The new sample preparation technique provides excellent resolution without the necessity for cryo temperatures. Figure 1 shows the comparison of the new method (Figure 1A) to cutting with a normal diamond microtomy knife (Figure 1B) and finally a razor blade (Figure 1C) on a piece of Southern Pine without polymer impregnation or embedding. The cross sections of the wood in both normal diamond knife microtomy and the razor blade cannot resolve the internal structure of the wood inside the cell wall. However, the new method provides a smooth sectioning of the wood which shows the middle lamella and the intracellular contents. Throughput is also increased since typical embedding methods may take from 12 hours to over 3 days to infiltrate samples.

Several analytical methods may now be utilized including electron microscopy, optical microscopy, and spectroscopy to probe polymer localization in the wood. Figure 2 demonstrates the utilization of some of these methods for probing the morphology and localization of polymer in the wood. By staining the wood with ruthenium tetroxide (to provide contrast) and using the new sample prep method, we can distinguish the untreated wood (Figure 2A, inset) from the polymer rich domain areas inside the wood (Figure 2B, inset), which have reacted with the ruthenium. A second example revolves around fluorescence microscopy. Since wood contains many phenolic compounds, it has an intrinsic autofluorescence in the green and blue wavelengths in the spectrum, and changes in these wavelength intensities may indicate areas with polymer penetration [3]. Figure 2A shows an untreated piece of wood with a polymer treated piece of wood in Figure $2 \mathrm{~B}$, where the differences in brightness at the same exposure are clearly evident.

In summary, the new preparation method has proven to be effective for studying polymer penetration into the components of wood without embedding. Analytical techniques which may have been limited due to embedding can be fully utilized to probe the internal polymer chemical interactions and localization. The application of this technique has contributed to the increased throughput of polymer 
formulations screening by decreasing the sample prep time, all which has translated to bringing promising formulations to customers at an accelerated rate.

References:

[1] C.E. Hsieh, M. Marko, J. Frank and C.A. Mannella: Electron tomographic analysis of frozenhydrated tissue sections. Journal of Structural Biology 138, pp. 63-73, 2002.

[2] J. R. McIntosh, "Electron Microscopy of Cells: A new beginning of a new century," The Journal of Cell Biology, vol. 153, pp. 25-32, 2001.

[3] N. J. Chaffey, Wood Formation in Trees: Cell and Molecular Biology Techniques. 2003, pp. 86-97.

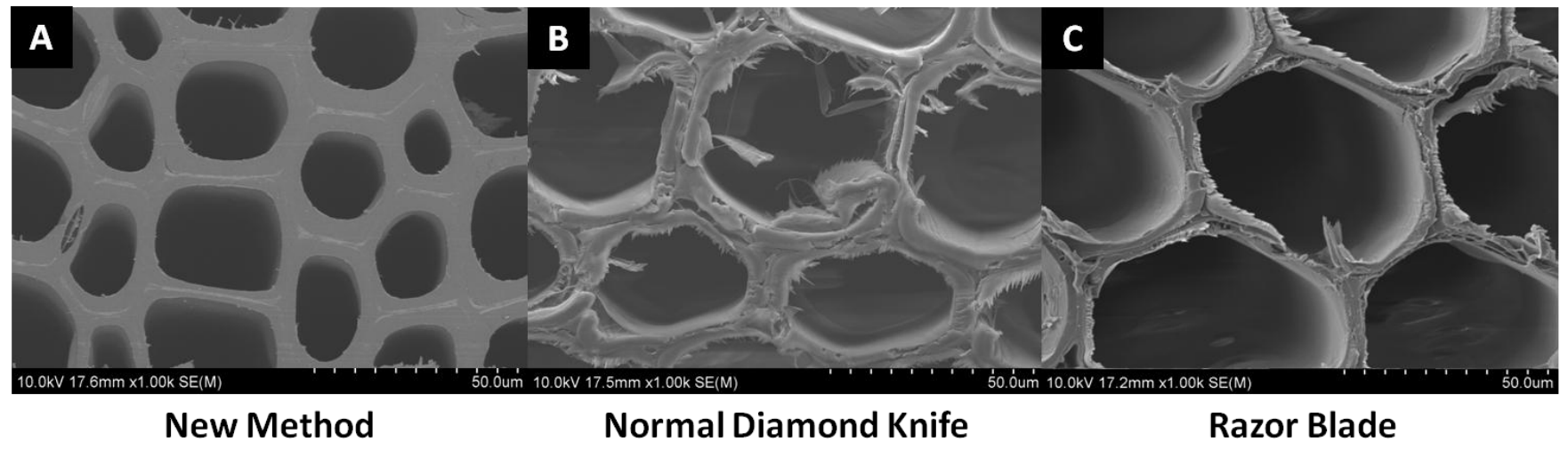

Figure 1. Scanning electron microscopy (SEM) image comparison of untreated wood block sections cut with the new method (A), a normal diamond knife (B), and a stainless steel razor blade (C). All images were taken at $1000 \mathrm{x}$ magnification.

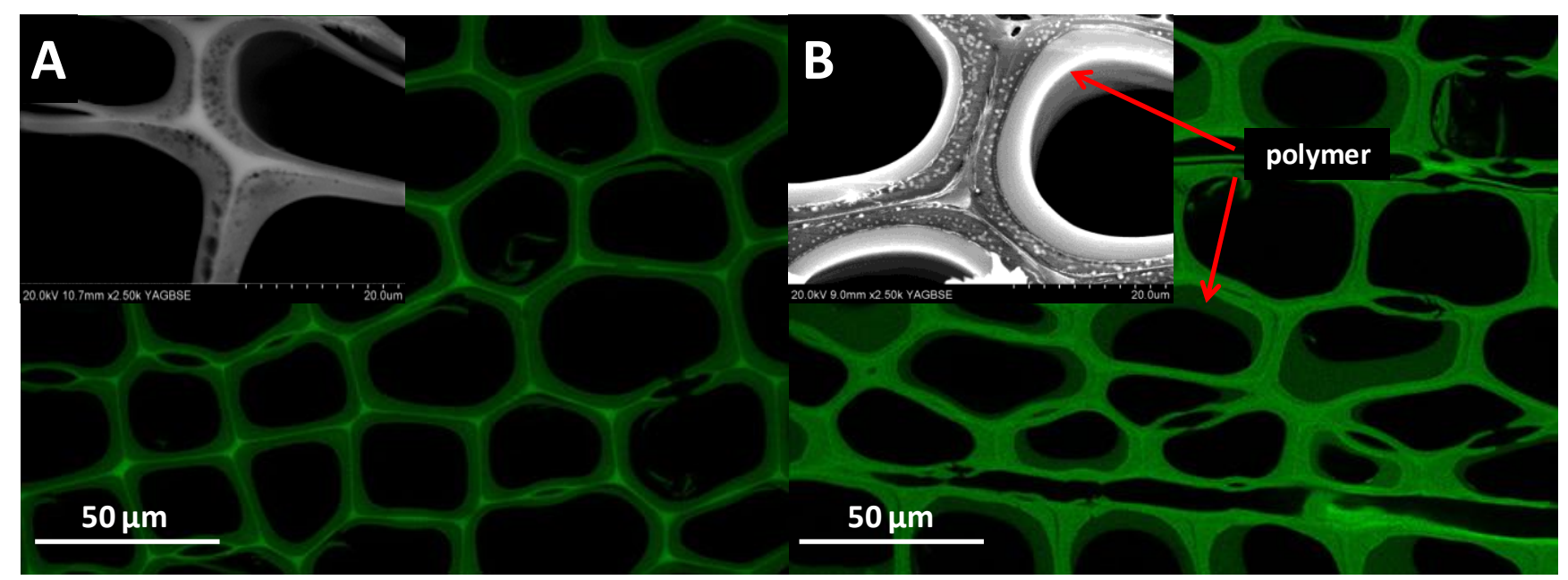

\section{Untreated}

Polymer Treated

Figure 2. A comparison of untreated wood (A) and polymer treated wood (B) sectioned using the new method, perpendicular to the grain of the wood. The SEM images (inset) show morphology differences from ruthenium staining between untreated and polymer treated wood. Corresponding fluorescence images are taken at the same exposure level of $100 \mathrm{~ms}$. 